\title{
In-between Bragg reflections: thermal diffuse scattering and vibrational spectroscopy with $\mathrm{X}$-rays
}

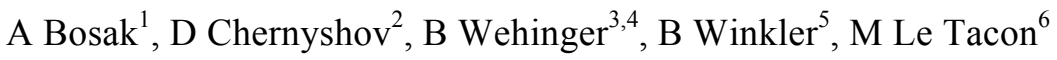 \\ and M. Krisch ${ }^{1}$
}

${ }^{1}$ ESRF - The European Synchrotron, 71, Avenue des Martyrs, F-38000 Grenoble, France

${ }^{2}$ Swiss-Norwegian Beamlines at European Synchrotron Radiation Facility, Grenoble, France

${ }^{3}$ Department of Quantum Matter Physics, University of Geneva, 24, Quai Ernest, Ansermet, CH-1211 Geneve, Switzerland

${ }^{4}$ Laboratory for Neutron Scattering and Imaging, Paul Scherrer Institute,

CH-5232, Villigen PSI, Switzerland

${ }^{5}$ Geowissenschaften, Goethe-Universität, Altenhoeferallee 1, D-60438 Frankfurt a.M., Germany

E-mail: bossak@esrf.fr

In the last decade diffuse scattering studies re-gained their place in the domain of lattice dynamics studies. The use of thermal diffuse scattering becomes particularly efficient when coupled with vibrational spectroscopy, where inelastic x-ray scattering can be advantageous compared to inelastic neutron scattering, and state-of-the-art ab initio calculations. We present a brief summary of the experimental and theoretical background, give an overview of the principle experimental implementations, and discuss a representative set of examples of such a combined approach.

PACS: 61.05.cp, 63.20.-e, 63.20.dk. 
In-between Bragg reflections: thermal diffuse scattering and vibrational spectroscopy with X-rays

\section{Introduction}

Since the early days of x-ray diffraction it was realized that besides sharp diffraction spots diffuse intensity is present and that this due to lattice vibrations, correlated disorder, defects or combinations thereof. The distribution of inelastic x-ray scattering by thermally populated phonons in reciprocal space carries information on the lattice dynamics [1]. This scattering reduces the intensity of Bragg spots, parameterized as Debye-Waller factors in crystal structure analysis, and substantially increases the intensity of the "background" [2]. Historically, phonon dispersion was first determined from thermal diffuse scattering (TDS) [3]; however, the potential of TDS remained largely unexploited and lattice dynamics studies were predominantly conducted using inelastic neutron (INS) and inelastic xray scattering (IXS). Recently, the advent of third generation synchrotron sources with their outstanding brilliance, in conjunction with the development of fast large area detectors has led to a revival of TDS studies [4,5].

INS instruments evolved from single analyzer triple-axis machines to multianalyzer setups and flat cone type devices allowing the mapping of 2D sections of reciprocal space. Time-of-flight-based INS spectrometers can provide 4D (Q-E) datasets on a reasonable timescale, providing an enormous gain. IXS instruments are based on the multianalyzer concept, and operate close to the practical limits of $\mathrm{x}$ ray fluxes - so the biggest breakthrough can be expected rather from the use of combined techniques (IXS+TDS, IXS+ab initio) than from gradual instrumental improvements.

From the intensities of inelastic scattering only limited information on the phonon eigenvectors is available [6]. Therefore, for a quantitative analysis of the dispersion relations it is necessary to introduce a model of interatomic interactions (the force matrix) which reproduces the eigenvalues, which, however, requires special care when studying phonons at low symmetry points in the Brillouin zone. Alternatively one may compare experiment with ab initio calculations, and, due the advent of mature DFT-based codes in conjunction with the availability of powerful computers, this is currently the most efficient approach.

TDS patterns naturally encode the information on the eigenvectors, i.e. if the model reproduces such patterns reasonably well, it means that both eigenvectors and eigenfrequencies are well reproduced. At the same time the energy scale cannot be retrieved directly - by the very nature of energyintegrating measurement. Nevertheless, in the specific cases TDS measurements may provide sufficiently constraining information to make the inelastic scattering experiment non-necessary. A fast mapping of reciprocal space, with or without modelling, allows the identification and localization of anomalies - through the manifestation of distinct intensity changes - which can then be further explored in detail by inelastic scattering experiments. 
In-between Bragg reflections: thermal diffuse scattering and vibrational spectroscopy with X-rays

In the following we summarize the theoretical background, recall the experimental techniques and their implementations and present the results of a representative set of benchmark systems of such combined approach:

- $\mathrm{SiO}_{2}$ polymorphs $\alpha$-cristobalite, $\alpha$-quartz and coesite:

- $\beta$ and $\gamma$ tin polymorphs

- Underdoped $\mathrm{YBa}_{2} \mathrm{Cu}_{3} \mathrm{O}_{7-\mathrm{x}}$ :

\section{Experimental techniques}

All very high resolution IXS instruments are based on the concept of the triple-axis spectrometer as developed by Brockhouse for INS [7]. The three axes comprise the very high energy resolution monochromator (first axis), the sample goniometry (second axis) and the crystal analyzer (third axis). A detailed account of the instrumental developments and two different types of very high energy resolution monochromators - backscattering and nested channel-cuts - is given in [8].

The requirement of momentum and energy conservation impose that:

$$
\begin{gathered}
\mathbf{Q}=\mathbf{k}_{i}-\mathbf{k}_{f} \\
E=E_{i}-E_{f}
\end{gathered}
$$

In order to reach sufficiently large momentum transfers to cover several Brillouin zones, X-rays with an energy of at least $\sim 10 \mathrm{keV}$ need to be employed. The typical energies of phonons $(E)$ are in the meV range, hence $E_{f} \approx E_{i}$, and the momentum transfer is only dependent on the incident wave vector (energy) and the scattering angle $2 \theta$ :

$$
Q=2 k_{i} \sin (\theta)
$$

Typically the scattered photons are energy analyzed by a spherical perfect silicon crystal analyzer, operated in Rowland geometry, and at a Bragg angle close to $90^{\circ}$. Most of the IXS spectrometers are equipped with more than one analyzer so that spectra at several momentum transfers can be recorded simultaneously.

Recently a conceptually new spectrometer featuring a spectral resolution function with steep, almost Gaussian tails, sub-meV bandwidth and improved momentum resolution was reported [9]. It combines flat crystal angular dispersive X-ray optics with collimating and focusing curved mirrors.

Energy scans are conveniently done by changing the lattice constant via the temperature $T: \Delta E / E=$ $\Delta d / d=\alpha(\mathrm{T}) \Delta T$, where $\alpha(\mathrm{T})=\alpha_{0}\left(\mathrm{~T}=22.5^{\circ} \mathrm{C}\right)+\beta \Delta \mathrm{T}\left[\mathrm{K}^{-1}\right]$ is the thermal expansion coefficient of silicon, equal to $2.58 \times 10^{-6} \mathrm{~K}^{-1}$ at ambient temperature [10]. 


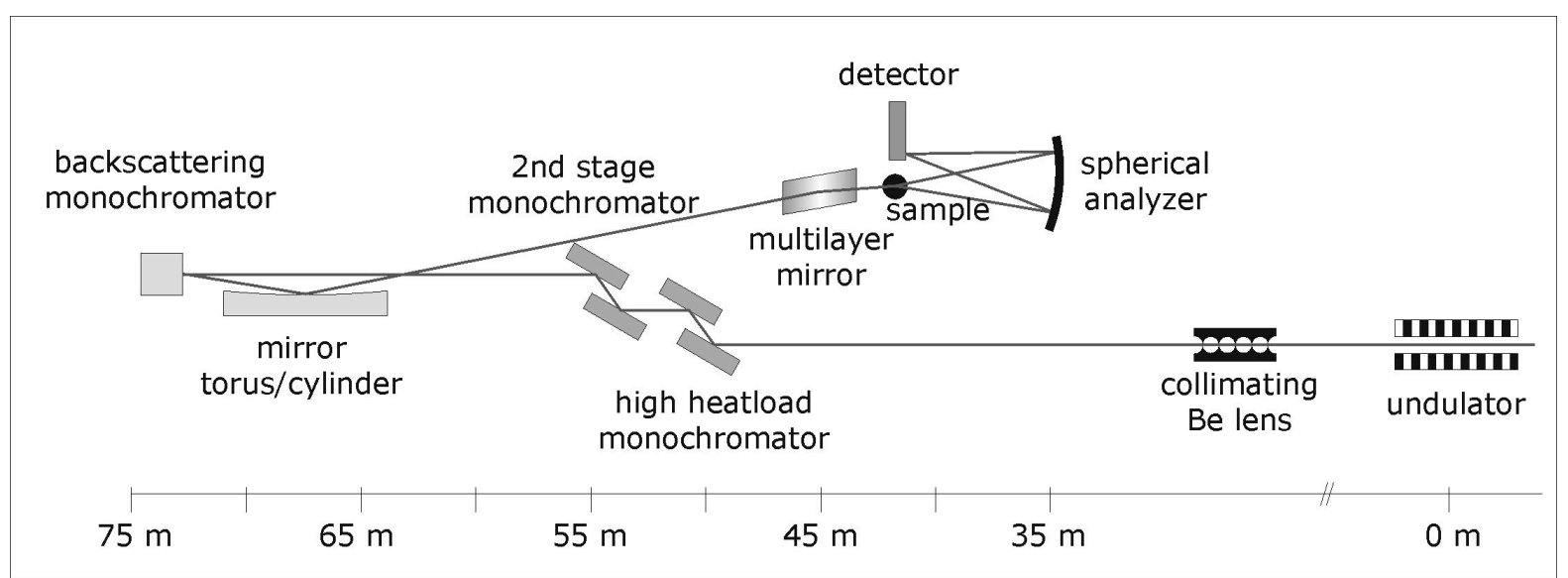

Figure 1. Schematic layout of the inelastic scattering beamline II (ID28) at the European Synchrotron Radiation Facility.

In order to obtain an energy step of about one tenth of the energy resolution, i.e., $\Delta E / E \sim 10^{-9}$, it is necessary to control the monochromator crystal temperature with a precision of about $0.5 \mathrm{mK}$ [11]. The scheme of the ID28 ESRF instrument layout is given in figure 1.

No such extreme monochromatization is necessary for diffuse scattering studies, and the use of a standard monochromator is generally sufficient. An example of a suitable undulator beamline is ID29 at the ESRF, which is optimized for protein crystallography, where a silicon $\left(\begin{array}{lll}1 & 1 & 1\end{array}\right)$ channel-cut monochromator is used, providing an energy range from $\sim 6$ to $\sim 20 \mathrm{keV}$. Several diffuse scattering experiments presented in this article were performed on this beamline. The optical layout of beamline is shown in figure 2. More details can be found elsewhere [12]. Conceptually, diffuse scattering experiment is similar to the "normal" diffraction studies, but with more severe requirements for the crystal quality and hardware. High photon flux, large dynamic range and low noise for the detector, and low signal contamination from the sample environments are the principal issues. Energy discrimination feature, available for hybrid pixel detectors, is extremely useful for the suppression of fluorescence contribution.

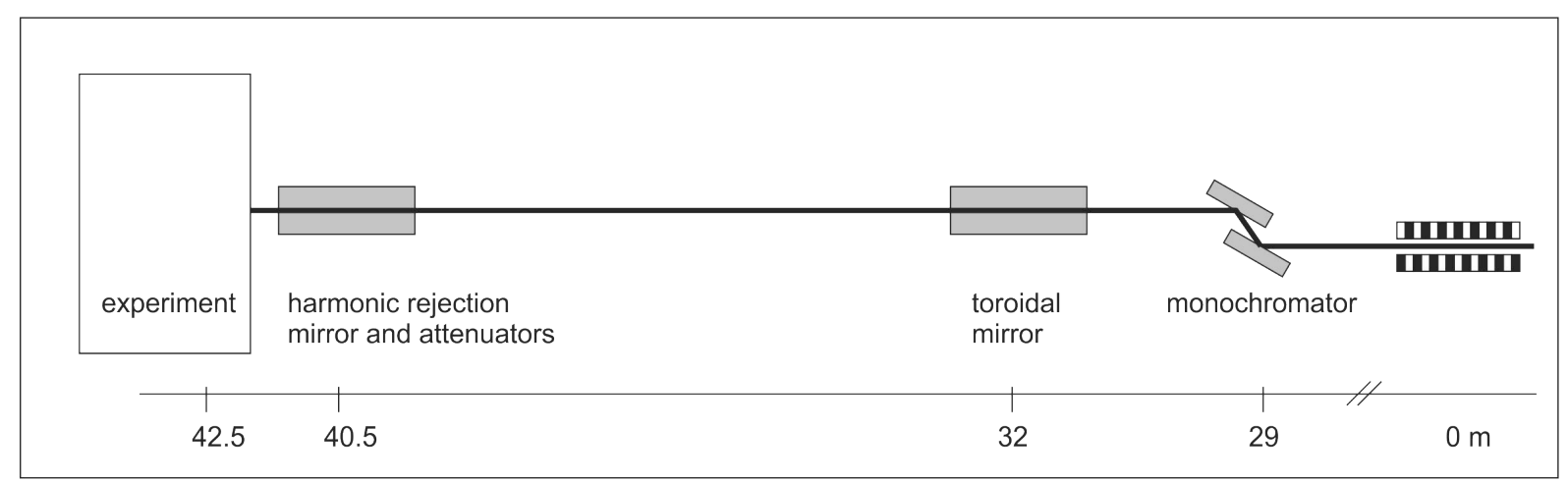

Figure 2. Schematic optical layout of beamline ID29 at the European Synchrotron Radiation Facility. 
In-between Bragg reflections: thermal diffuse scattering and vibrational spectroscopy with X-rays

In the pioneering work of Holt [4] the thermal diffuse scattering from silicon was recorded for a set of high-symmetry orientations in order to fit the data by a Born-von Kármán model. A rather high photon energy $(28 \mathrm{keV})$ and an image plate detector with rather long readout time was used. Nowadays, hybrid pixel photon counting detectors [13] are the solution of choice thanks to the extremely low intrinsic noise, large dynamic range, ability to suppress the fluorescent background, and very short (milliseconds) readout time, allowing shutterless data collection. Photon energies are limited to $25-30 \mathrm{keV}$ due to the dramatic decrease of the efficiency of silicon-based sensors for higher energies. High-Z hybrid pixel photon counting detectors open the way towards efficient measurements at much higher energies [14], but their commercialization has started only recently. Generally, the choice of the optimal energy is a trade-off, taking into account the sample absorption, focal size, fluorescence suppression and available equipment.

All the results presented below were obtained with PILATUS detectors [13], at beamline X06a at the Swiss Light Source, beamline BM01A at SNBL at the ESRF, and beamlines ID23-1 and ID29 at the ESRF. Crystals with a thicknesses smaller or comparable to the absorption length at a given wavelength were mounted on a rotation stage, and diffuse scattering patterns were recorded with PILATUS 6M or $2 \mathrm{M}$ (BM01A) pixel detectors with an increment of $\Delta \varphi=0.1^{\circ}$ over an angular range of $360^{\circ}$. Orientation matrix refinement and preliminary reciprocal space reconstructions were performed using the CrysAlis software package [15]. For the final reciprocal space reconstructions we applied corrections for polarization (pure linear, $\varphi$ is the angle between the plane of polarization of the incident beam and the scattering plane)

$$
P=\sin ^{2}(\varphi)+\cos ^{2}(\varphi) \cos ^{2}(2 \theta)
$$

and geometrical correction associated with the planar projection

$$
D=\cos ^{-3}(2 \theta)
$$

The reconstructed planes were averaged with their symmetrically equivalent orientations employing the Laue symmetry of the sample, thus improving the signal-to-noise ratio and removing the gaps between the individual detector elements. 
In-between Bragg reflections: thermal diffuse scattering and vibrational spectroscopy with X-rays

\section{Theoretical formalism}

We use the following notations: $\omega_{j}(\mathbf{q})=\hbar^{-1} E_{j}(\mathbf{q})$ - frequency of mode $j(\mathrm{j}=1, \ldots 3 \mathrm{n}, \mathrm{n}$ is the number of atoms in the primitive unit cell) at reduced momentum transfer $\mathbf{q}=\mathbf{Q}-\boldsymbol{\tau}$ ( $\boldsymbol{\tau}$ is the closest reciprocal lattice vector), $\boldsymbol{\sigma}_{d}^{j}-d$-site projected component of $3 N$-dimensional normalized eigenvector $\boldsymbol{\sigma}^{j}$ of the phonon mode $j$. We use the dynamical matrix $\mathbf{C}(\mathbf{q})$, which is Hermitean by construction and periodic: $\mathbf{C}(\mathbf{q}+\boldsymbol{\tau})=\mathbf{C}(\mathbf{q})$.

Assuming the validity of harmonic approximations [16], the general form of the dynamical structure factor $S(\mathbf{Q}, E)$ for single-phonon scattering takes the following form

$$
\begin{gathered}
S(\mathbf{Q}, E)=\sum_{j} G(\mathbf{Q}, j) F(E, T, \mathbf{Q}, j) \\
G(\mathbf{Q}, j)=\left|\sum_{d} f_{d}(\mathbf{Q}) e^{-W_{d}(\mathbf{Q})+i \mathbf{Q} \cdot \mathbf{r}_{d}}\left(\mathbf{Q} \cdot \boldsymbol{\sigma}_{d}^{j}(\mathbf{q})\right) M_{d}^{-1 / 2}\right|^{2}
\end{gathered}
$$

The thermal factor can be written as

$$
F(E, T, \mathbf{Q}, j)=\frac{1}{E_{j}(\mathbf{q})} \cdot\left[\left(<n\left(E_{j}(\mathbf{q}), T\right)>+1\right) \delta\left(E-E_{j}(\mathbf{q})\right)+<n\left(E_{j}(\mathbf{q}), T\right)>\delta\left(E+E_{j}(\mathbf{q})\right)\right](7)
$$

where the thermal occupation factor $<n(E, T)>=\left(\exp \left(\frac{E}{k_{B} T}\right)-1\right)^{-1}, f_{d}(\mathbf{Q})$ - atomic scattering factor of atom $d$ at the position $\mathbf{r}_{d}$, with mass $M_{d}$ and Debye-Waller factor $W_{d}(\mathbf{Q}) ; k_{B}$ - Boltzmann's constant; $\mathrm{T}$ - temperature. For neutron scattering the atomic structure factor is Q-independent. The anisotropic Debye-Waller factor can be computed for a given temperature as

$$
\begin{gathered}
W_{d}(\mathbf{Q})=\frac{1}{2} \mathbf{Q}^{T} \mathbf{U}_{d} \mathbf{Q} \\
\mathbf{U}_{d}=\frac{\hbar}{2 N M_{d}} \sum_{\mathbf{q}, j} \omega_{j}^{-1}(\mathbf{q})\left|\mathbf{\sigma}_{d}^{j}(\mathbf{q})\right|^{2} \operatorname{coth}\left(\hbar \omega_{j}(\mathbf{q}) / 2 k_{B} T\right)
\end{gathered}
$$

Here $\mathrm{N}$ is the number of wave vectors in the first Brillouin zone. The $\mathrm{x}$-ray intensity of first-order thermal diffuse scattering from a crystal containing $N$ atoms in the unit cell at temperature $T$ and momentum transfer $\mathbf{Q}$ can be written as [17]

$$
I(\mathbf{Q}) \propto \sum_{j=1}^{3 N} \frac{1}{\omega_{j}(\mathbf{q})} \operatorname{coth}\left(\frac{\hbar \omega_{j}(\mathbf{q})}{2 k_{B} T}\right)\left|\sum_{d=1}^{N} f_{d}(\mathbf{Q}) \exp \left(-W_{d}(\mathbf{Q})+i \mathbf{Q} \cdot \mathbf{r}_{d}\right)\left(\mathbf{Q} \cdot \boldsymbol{\sigma}_{d}^{j}(\mathbf{q})\right) M_{d}^{-1 / 2}\right|^{2}
$$


Note that the above expression contains both eigenvalues $\omega_{j}(\mathbf{q})$ and eigenvectors $\boldsymbol{\sigma}^{j}(\mathbf{q})$ of the dynamical matrix $\mathbf{C}(\mathbf{q})$.

This equation can be rewritten in the alternative form

$$
I(\mathbf{Q}) \propto \mathbf{Q}^{T} \cdot \sum_{j=1}^{3 N} \frac{1}{\omega_{j}} \operatorname{coth}\left(\frac{\hbar \omega_{j}}{2 k_{B} T}\right) \mathbf{Z}(\mathbf{Q}) \mathbf{P}(\mathbf{q}) \mathbf{Z}^{*}(\mathbf{Q}) \cdot \mathbf{Q}
$$

where $Z_{\alpha \alpha}(\mathbf{Q})=f_{[\alpha / 3]}(\mathbf{Q}) \exp \left(-W_{[\alpha / 3]}(\mathbf{Q})+i \mathbf{Q} \cdot \mathbf{r}_{[\alpha / 3]}\right) M_{[\alpha / 3]}{ }^{-1 / 2}$ and the momentum transfer vector is redefined in $3 \mathrm{~N}$-dimensional space merging the three-dimensional $\mathbf{Q} \mathrm{N}$ times as $\mathbf{Q}^{T}=\left(\begin{array}{lllllllll}Q_{x} & Q_{y} & Q_{z} & \ldots & \ldots & \ldots & Q_{x} & Q_{y} & Q_{z}\end{array}\right)$. Note that the matrix $\mathbf{Z}(\mathbf{Q})$ depends on the atomic coordinates and Debye-Waller factors only, and can therefore be obtained from a Bragg diffraction experiment.

Since the $\omega_{j}^{2}$ are the eigenvalues of the (Hermitean) dynamical matrix $\mathbf{C}(\mathbf{q})$, the intensity reads

$$
I(\mathbf{Q}) \propto \mathbf{Q}^{T} \cdot \mathbf{Z}(\mathbf{Q}) \mathbf{S}(\mathbf{q}) \mathbf{Z}^{*}(\mathbf{Q}) \cdot \mathbf{Q}
$$

where

$$
\mathbf{S}(\mathbf{q})=\frac{\hbar}{2 \sqrt{\mathbf{C}(\mathbf{q})}} \operatorname{coth}\left(\frac{\hbar \sqrt{\mathbf{C}(\mathbf{q})}}{2 k_{B} T}\right)=k_{B} T\left(\mathbf{C}^{-1}(\mathbf{q})+\frac{1}{12}\left(\frac{\hbar}{k_{B} T}\right)^{2} \mathbf{I}-\frac{1}{720}\left(\frac{\hbar}{k_{B} T}\right)^{4} \mathbf{C}(\mathbf{q})+\ldots\right)
$$

and I stands for a unit matrix of appropriate size. The first term in Eq. 8 is sufficient for the description if $\left(\frac{\hbar}{k_{B} T}\right)^{2}\|\mathbf{C}(\mathbf{q})\|<1[17]$. Obviously, for diffuse scattering of neutrons, where energy and momentum transfers are coupled, the above treatment is not valid.

Eq. 11 defines the so-called S-matrix introduced by M. Born in 1942 [18] and already contained in I. Waller's dissertation, as M. Born mentions. The S-matrix decomposition has been applied before for the analysis of Debye-Waller factors [19,20,21].

When computing scattering intensities from eigenvectors and eigenfrequencies, special care has to be taken in the definition of the dynamical matrix, i.e. whether the C- or D-notation is employed [5], and the convention used for the phase factor. 


\section{Case study: covalent framework structures of $\mathrm{SiO}_{2}$}

To a large extent, the work on silica polymorphs was motivated by the quest to provide an atomistic description for the Boson peak, an anomaly in the low energy part of the phonon density-of-states in glasses [22]. Considerable efforts were undertaken to link the properties of glasses to the properties of crystalline counter partners [23], and in this context the detailed knowledge of the lattice dynamics of different $\mathrm{SiO}_{2}$ frameworks is very important. Combined studies by diffuse scattering, inelastic scattering and ab initio calculations were performed for three polymorphs, namely $\alpha$-cristobalite [24], $\alpha$-quartz [25] and coesite [26].

Some experimental parameters are given in Table 1. Lattice dynamical calculations were performed within density functional perturbation theory (DFPT) implemented in CASTEP [27,28]. The local density approximation and norm-conserving pseudopotentials were employed. TDS intensity calculations followed the well-established formalism [5,29] with anisotropic Debye-Waller factors derived from the lattice dynamics model, as explained in part 2.

Table 1. Experimental parameters for the silica polymorphs measurements.

\begin{tabular}{|c|c|c|c|}
\hline & $\alpha$-cristobalite & $\alpha$-quartz & coesite \\
\hline Source & Natural, Ellora Caves* & Synthetic, commercial & $\begin{array}{l}\text { Hydrothermal growth } \\
\qquad[30]\end{array}$ \\
\hline sample & $\begin{array}{c}\sim 0.2 \mathrm{~mm} \text { octahedron } \\
\text { twinned }\end{array}$ & $\varnothing 1 \mathrm{~mm}$ needle & $\begin{array}{c}0.1 \times 0.2 \times 0.3 \mathrm{~mm}^{3} \\
\text { twinned }\end{array}$ \\
\hline DS experiment & $\begin{array}{l}\text { ESRF, ID29 } \\
\lambda=0.700 \mathrm{~A}\end{array}$ & $\begin{array}{l}\text { SLS, X06SA } \\
\lambda=0.708 \mathrm{~A}\end{array}$ & $\begin{array}{l}\text { ESRF, ID29 } \\
\lambda=0.700 \mathrm{~A}\end{array}$ \\
\hline IXS experiment & \multicolumn{3}{|c|}{$\begin{array}{c}\text { ESRF, ID28, } \lambda=0.6968 \mathrm{~A} \text {, energy resolution } 3 \mathrm{meV} \text {, momentum resolution } \\
\qquad 0.25 \mathrm{~nm}^{-1} \times 0.75 \mathrm{~nm}^{-1}\end{array}$} \\
\hline publication & {$[24]$} & {$[25]$} & {$[26]$} \\
\hline
\end{tabular}

* Natural $\alpha$-cristobalite crystals from the Ellora Caves, Hyderabad, India were kindly made available by the Harvard Mineralogical Museum collection 
In-between Bragg reflections: thermal diffuse scattering and vibrational spectroscopy with X-rays

\section{1. $\alpha$-cristobalite}

So far, the lattice dynamics of $\alpha$-cristobalite has been investigated by inelastic neutron, Brillouin and Raman scattering. The unavailability of large single crystals prevented the measurements of phonon dispersion curves by neutron scattering.

In our experiment, one major twin [31] and some mosaic spread $\left(<1^{\circ}\right)$ were observed. One domain could be isolated for the experimental study thanks to the small x-ray beam size, and only a small contribution from the other twins was apparent. The experimental intensity distributions show signatures of the mosaic spread, which appear as arc-like features. A complex distribution of diffuse scattering is noticeable, mainly in the shape of streaks along $\langle 100\rangle,\langle 110\rangle,\langle 101\rangle$ and $\langle 112\rangle$ directions, as can be appreciated from high symmetry sections in figure 3a-c. The diffuse scattering along $<1 \quad 10>$ and $<101>$ is in agreement with the prediction of the rigid unit modes model [32]. The simulated intensity distribution reproduces all the essential features of the experiment; figure $3 \mathrm{~d}$ illustrates the local 3D distribution of the intensity as modelled in the proximity of the (332) node. 

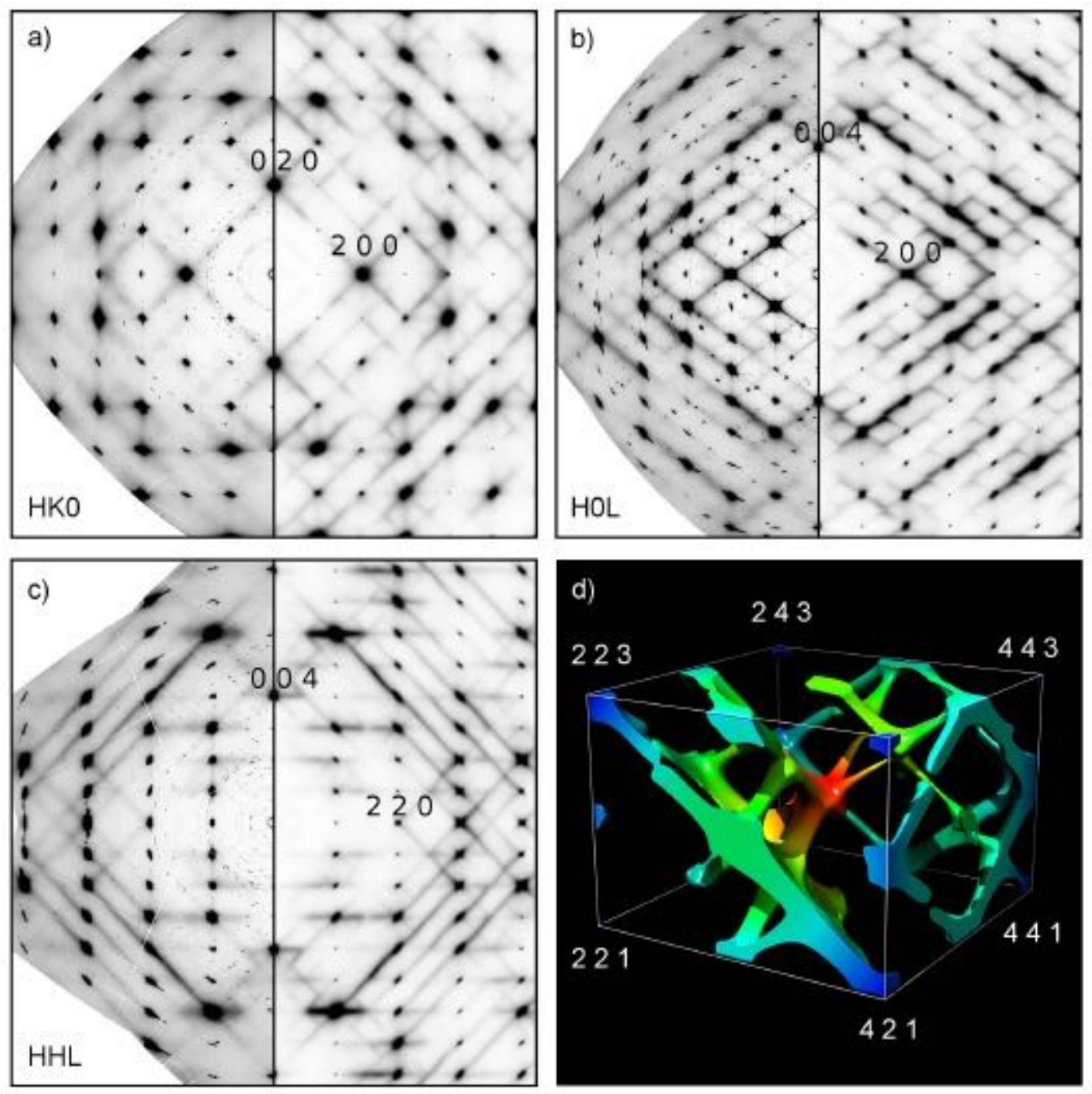

Figure 3. $(\mathrm{a}-\mathrm{c})$ Experimental diffuse scattering (left part of individual panels) and calculated (right part of individual panels) TDS intensity distribution of single crystal $\alpha$-cristobalite in the indicated reciprocal space sections. (d) Calculated iso-intensity distribution of TDS around ( $\left.\begin{array}{lll}3 & 3 & 2\end{array}\right)$. The colours denote the distance from the center. Taken from [24]. Color online.

Some regions of interest, associated to strong DS and thus soft phonons, were studied in more details by IXS. IXS intensity maps (figure 4) further validate the model in terms of phonon energies. Applying a uniform scaling factor of 1.039 to the calculated phonon energies both energies and intensity of the phonon excitations match. The scaling factor was determined from the vibrational density of states measured on the polycrystalline sample (see [24] for details). The minima of the lowest energy phonon branch are slightly underestimated, while the overall agreement remains very reasonable. 
a)

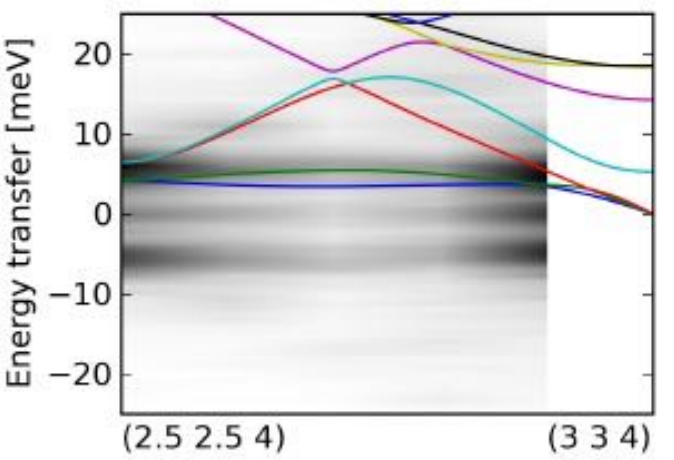

b)

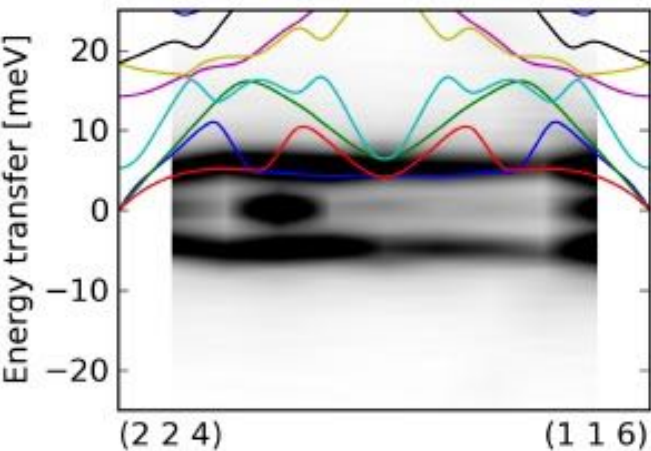

c)

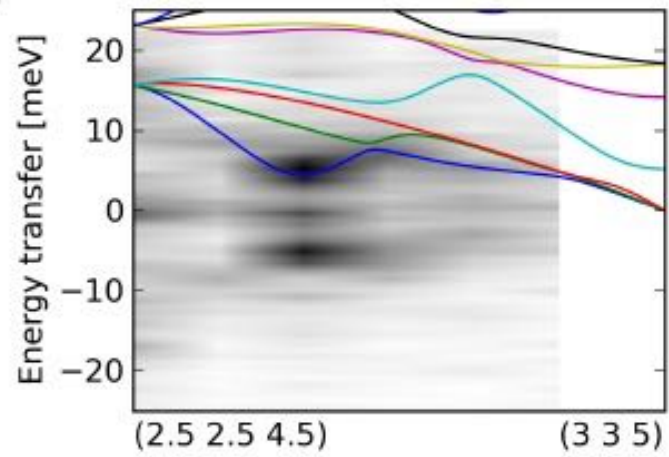

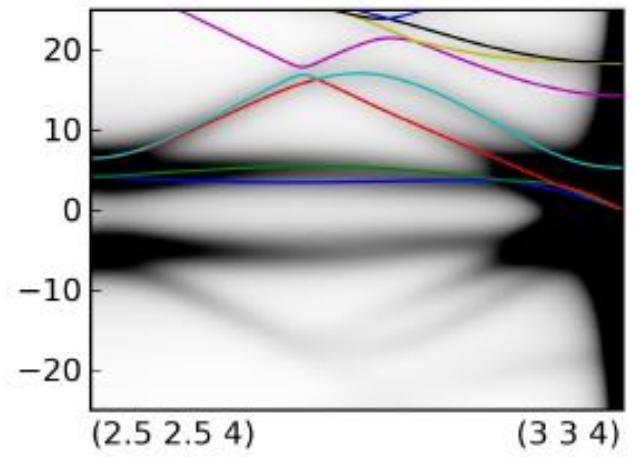
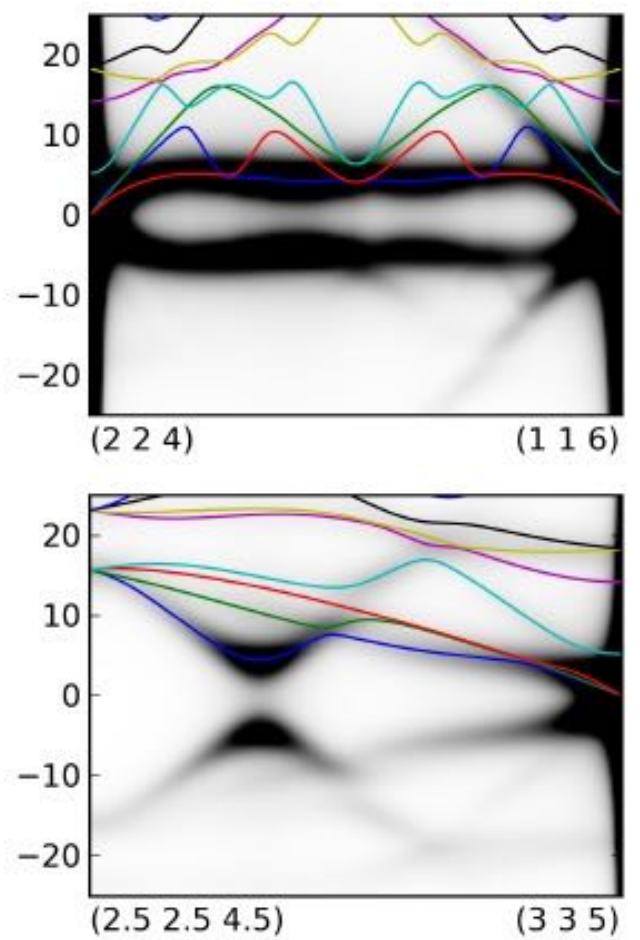

Figure 4. Experimental IXS intensity maps (left panels) of $\alpha$-cristobalite together with theoretical intensity maps (right panels) along $<110>$ (a), $<112>$ (b) and $<111>$ (c). The theoretical dispersion relations are traced as lines. Taken from [24]. Color online.

\section{2. $\alpha$-quartz}

The lattice dynamics of quartz has been extensively studied, but a complete lattice dynamics description, in which not only the phonon dispersion curves, but also the phonon eigenvectors for arbitrary momentum transfers are established, was still missing. An impressive study of the phonon eigenvectors of quartz at the M point was performed by INS [33], but in view of the relative complexity of the structure - with 9 atoms per elementary cell and 27 phonon branches - it is unlikely that such a study will be performed for anything but a very limited number of reciprocal lattice points and modes. Ab initio calculations of the phonon dispersion curves of quartz are generally in very good 
agreement with experimentally observed frequencies, which offered us an occasion to provide an overall validation of the lattice dynamics, going beyond high-symmetry points and directions.

Compared to a-cristobalite, the diffuse features are more complex; they cannot be reduced to a set of diffuse streaks anymore (figure 5). Remarkably, even the tiniest details are reproduced by the DFPT calculations. The validity of the calculations was further confirmed exploring the inelastic scattering in selected directions (figure 6), including those of low symmetry, and measurements of powder inelastic spectra (not shown here, see [25]).

It is interesting to note, that rather smooth diffuse features can correspond to the pile-up of many branches, with apparently irregular intensity distribution between them as seen in figures $6 \mathrm{a}$ and $6 \mathrm{c}$.

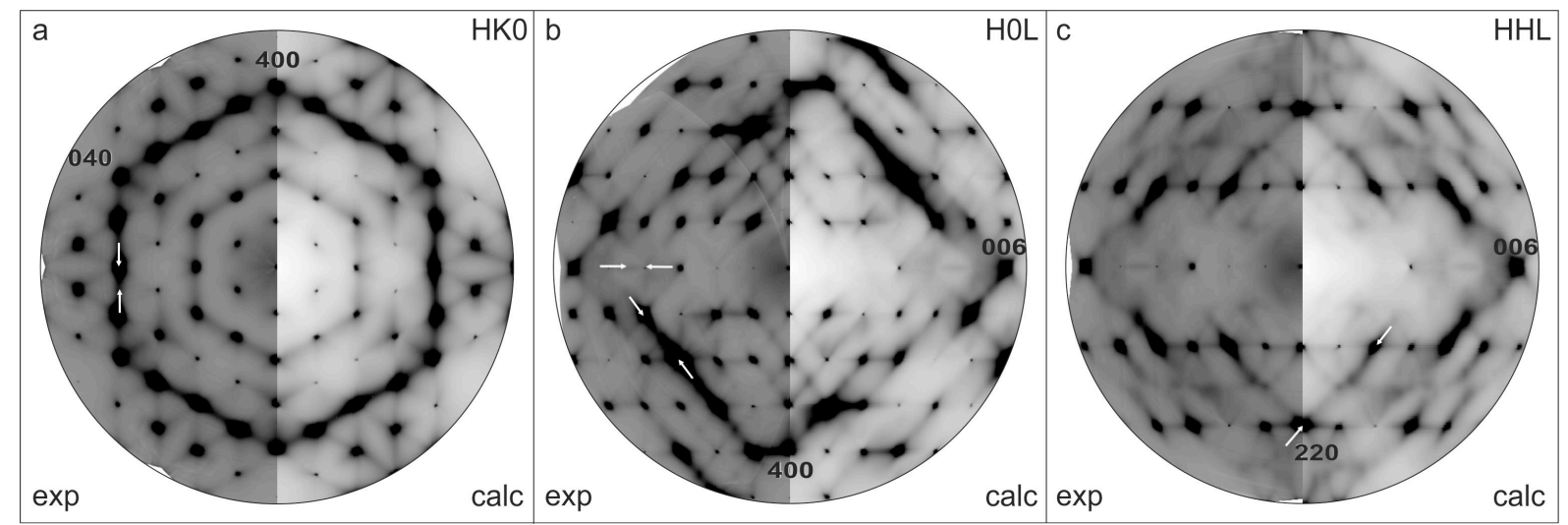

Figure 5. Experimental and calculated TDS patterns of $\alpha$-quartz in high-symmetry reciprocal space sections HK0 (a), H0L (b) and HHL (c). Experimental artifacts due to non-uniform absorption are visible. White arrows denote the sections for which IXS (E-Q) maps were recorded. Adapted from [25]. 

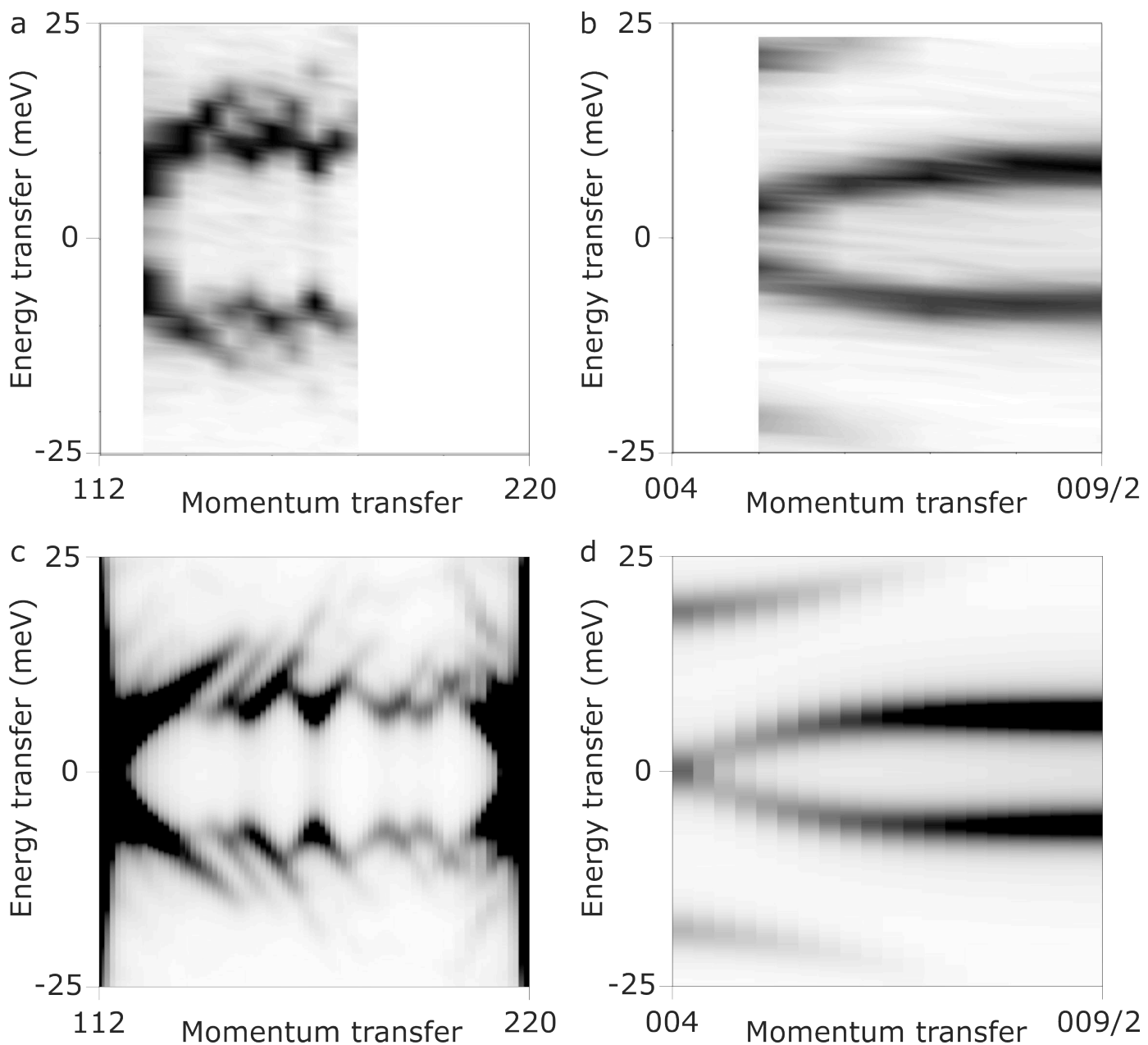

Figure 6. Experimental $(a, b)$ and calculated (c-d) (E-Q) intensity maps for $\alpha$-quartz. Panel (a) can be directly compared to (c), and panel (b) can be compared to (d). Adapted from [25].

\subsection{Coesite}

Coesite is the highest density crystalline polymorph of silica in which the silicon atoms are tetrahedrally coordinated. The structure is monoclinic, but metrically nearly hexagonal. It was first synthesized in 1953 at high pressure and high temperature, and was later found in sandstone of the Arizona Barringer crater, leading to the general acceptance of the impact cratering theory and to important implications for the recognition of meteorite impact craters in quartz-bearing geologic formations [34].

Diffuse scattering patterns are very structured, and they can be rationalized only if pseudohexagonal twinning is taken into account (figure 7) with two twins with the occupancy ratio about 3:1. 

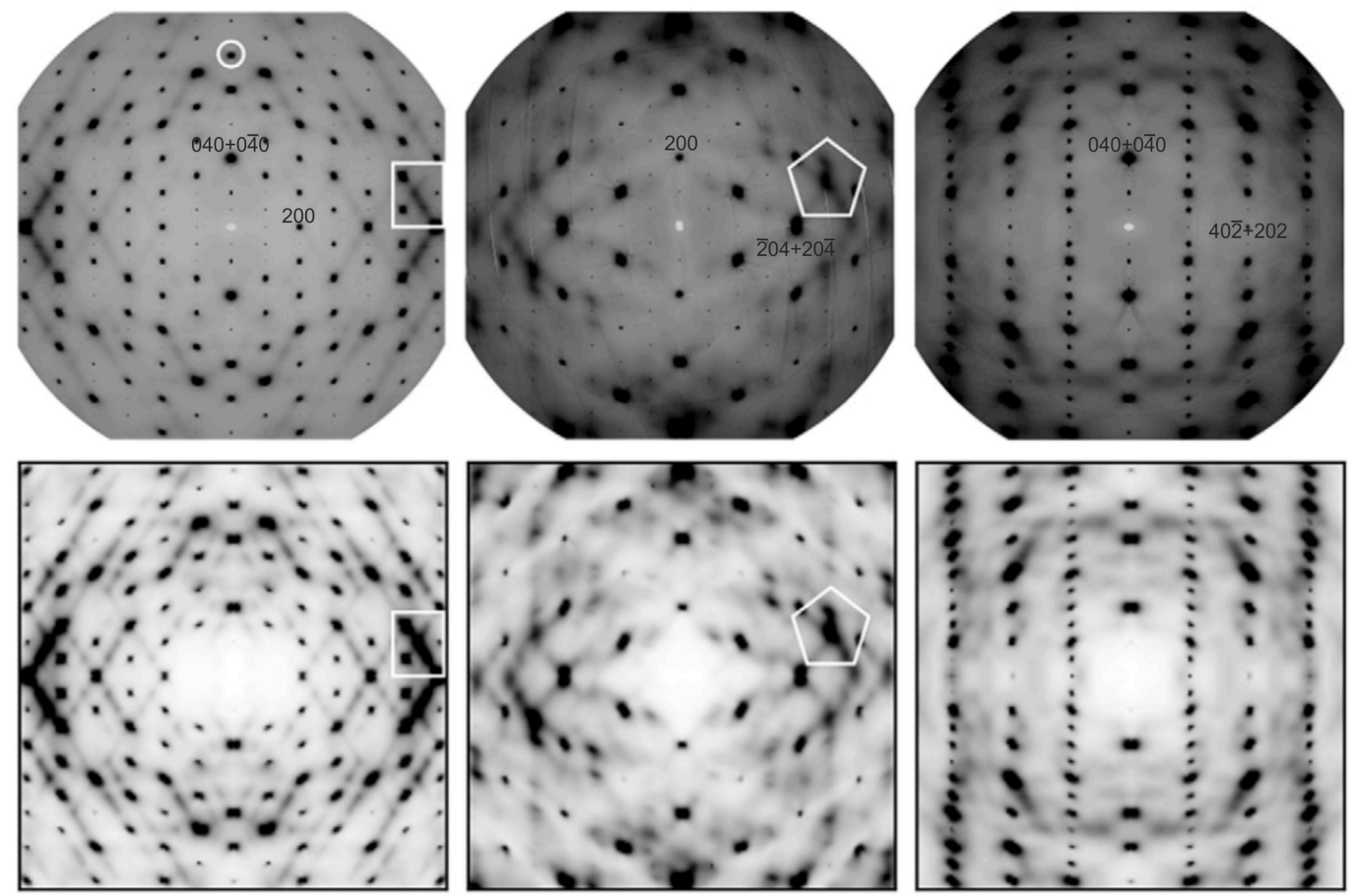

Figure 7. Experimental (first row) and calculated (second row) diffuse scattering intensity distributions of coesite in high-symmetry reciprocal space sections. The absolute intensity is scaled to optimise the visualization of the diffuse features. The calculated intensity distributions in the second row are created by a weighted superposition of the two twins $(\sim 3: 1)$. The diffuse features marked by a circle and a polygon were selected for an IXS study. The intense features highlighted by a rectangle and a polygon are guides for the comparison of experimental and calculated diffuse scattering. Taken from [26].

As for cristobalite, only a modest scaling of the energy had to be applied in order to reconcile the experiment and $a b$ initio calculations. After scaling the calculated energies by 1.045 the theoretical spectra reproduce quite well both the position and intensity of the phonons (figure 8). The scaling factor, appropriate for the entire energy scale, was - as above- determined from the VDOS measured on polycrystalline samples. 

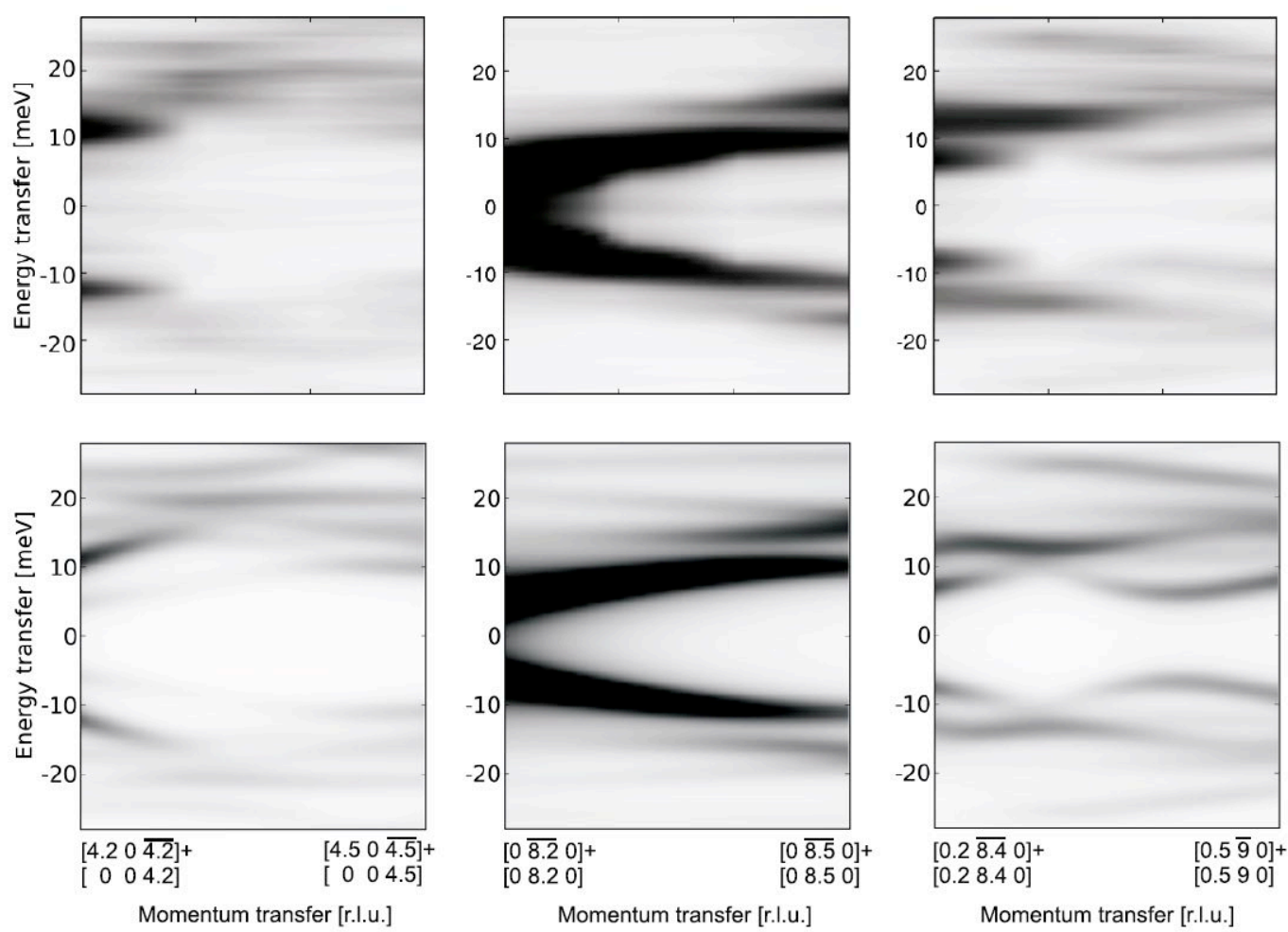

Figure 8. Experimental IXS intensity maps (first row) from coesite together with theoretical intensity maps (second row) along the indicated directions. Taken from [26].

In all the above cited examples of $\mathrm{SiO}_{2}$ frameworks the results were very satisfactory. The full description of the lattice dynamics at arbitrary momentum transfer allows for an investigation of the phonon energy landscape. In particular, the critical points responsible for Van Hove singularities can be localized - potentially interesting for studies addressing the nature and origin of the boson peak. The eigenvectors and the dispersion for the branch contributing most to the first peak in the vibrational density of states can be assigned. Not very surprisingly, the critical points are located at the zone boundary; the vibration contributing the most is mainly driven by oxygen displacements, corresponding to a tetrahedron tilt accompanied by a small distortion.

In summary, we were able to provide accurate models of the lattice dynamics of $\alpha$-cristobalite, $\alpha$ quartz, coesite and - for a different type of silicon coordination - stishovite [35]. The calculations for these polymorphs can be extended to high pressures in a straightforward fashion and allow the derivation of elastic and thermodynamic properties. Such an extension potentially builds the basis for understanding the compression mechanism and phase stability at geophysically relevant conditions. The prediction of the lattice dynamics should also be possible within the employed calculation scheme for the high pressure phases of cristobalite [36] as well as for the recently discovered highpressure silica polymorphs seifertite and others [37]. For cristobalite the knowledge of the lattice dynamics at high pressures is in particular important for understanding the pressure evolution of its auxetic behaviour [38] as well as the phase stability and elasticity of its high pressure phases. 
In-between Bragg reflections: thermal diffuse scattering and vibrational spectroscopy with X-rays

\section{Case study: tin polymorphs}

Metallic tin polymorphs were studied as a benchmark case for metallic systems of medium structural complexity. Tin crystallizes in a body-centred tetragonal lattice (space group $\mathrm{I}_{1} / \mathrm{amd}$ ) at ambient conditions, this polymorph is known as white tin $(\beta-\mathrm{Sn})$. Despite the fact that the stability range of white tin lies between 291 and $\approx 450 \mathrm{~K}$ it can be supercooled far below the transition temperature, maintaining the crystal structure. Below $\approx 4.2 \mathrm{~K}$ it becomes a type-I superconductor which can be described in the frame of BCS theory, indicating strong electron-phonon coupling [39]. Alloying tin with indium results in a substitutionally disordered crystal with a primitive hexagonal lattice containing one atom per unit cell, called $\gamma$-tin. It is a convenient model system in the study of lattice dynamics and electron-phonon interactions, because its phonon dispersion relations consist only of acoustic branches and it is stable at ambient conditions. The "true" $\gamma$-phase of pure tin is orthorhombic and differs thus slightly from the primitive hexagonal lattice. It is stable between $\approx 450 \mathrm{~K}$ and the melting point of tin $(505 \mathrm{~K})[40]$.

First Laue photographs of white tin showing a 'diffuse background with regions of maximum intensities' were published in 1943 [41] and 'considered in the light of thermal theory' in 1946 [42]. Elastic coefficients were derived from the diffuse features in $1955\left[{ }^{43}\right]$, followed by inelastic neutron scattering study of the phonon dispersion relations $[44,45,46]$ along high symmetry directions. The lattice dynamics of $\gamma$-tin (stabilized by indium addition) was also studied by INS [47]. In [48] we investigate the lattice dynamics of the metallic tin polymorphs employing a combination of TDS, IXS and DFPT in order to obtain the full description of the lattice dynamics at arbitrary momentum transfer.

Some experimental parameters are given in Table II. First principles lattice dynamics calculations were performed with the CASTEP package $[27,28]$ at $0 \mathrm{~K}$. The acoustic sum rule correction was applied to the calculated dynamical matrix in order to account for translational invariance with maximal correction of $2 \mathrm{meV}$ at $\Gamma$ point. More details are given in [48]. 
In-between Bragg reflections: thermal diffuse scattering and vibrational spectroscopy with X-rays

Table 2. Experimental parameters for tin polymorphs measurements.

\begin{tabular}{lcc}
\hline \multicolumn{1}{c}{$\beta$-tin } & $\gamma$-tin \\
\hline sample & $\varnothing 0.1 \mathrm{~mm}$ needle Sn & $\varnothing 0.1 \mathrm{~mm}$ needle $\mathrm{Sn}_{0.8} \mathrm{In}_{0.2}$ \\
DS experiment & ESRF, ID29 \\
& ESRF, ID29 & $\lambda=0.700 \mathrm{~A}$ \\
$\lambda=0.700 \mathrm{~A}$ & \\
IXS experiment & ESRF, ID28, $\lambda=0.6968 \mathrm{~A}$, energy resolution $3 \mathrm{meV}^{2}$ momentum \\
publication & {$[48] \quad[48]$} \\
\hline
\end{tabular}

The diffuse features, observed in white tin, are atypical for a simple metal (figure 9). One of the most striking features was the strong asymmetry of diffuse clouds in HK2n+1 reciprocal space sections. It is most pronounced around the (211) reflection. The IXS investigation, in agreement with the calculation, shows a drastic change of spectral weight between the two branches $\Delta_{3}$ and $\Delta_{4}$ - when the sign of the reduced momentum transfer $\mathrm{q}$ is changed - resulting in an energy shift of the envelope function (figure 10) - and q/-q asymmetry of the diffuse features. The $\Delta_{4}$ optic branch behaves in a similar way, being almost completely suppressed on one side only. 

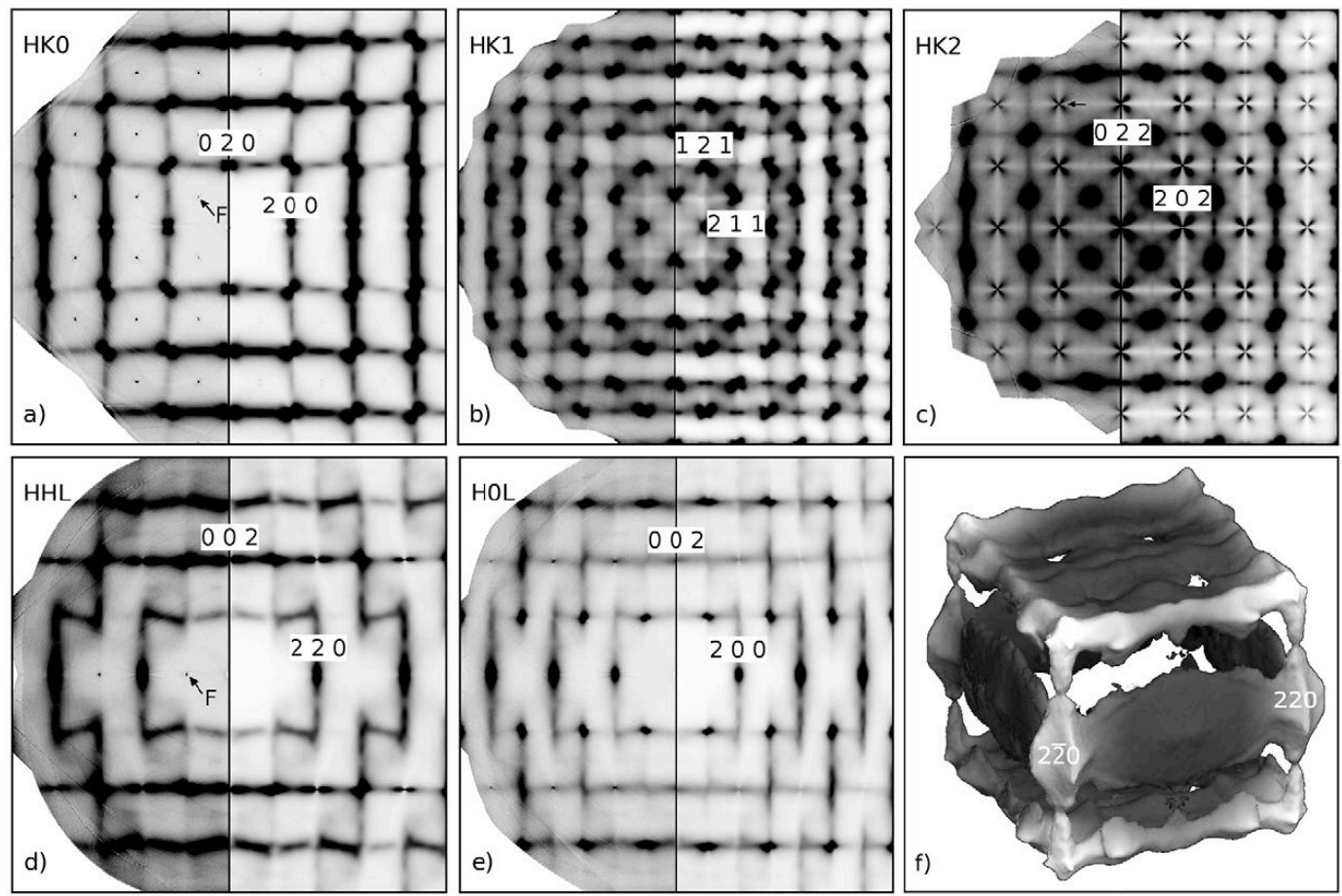

Figure 9. (a)-(e) Experimental diffuse scattering (left part of individual panels) and calculated (right part of individual panels) TDS intensity distribution of $\beta$-tin in the indicated reciprocal space sections. Note the almost forbidden reflections in (a), (c) and (d) (arrows labelled F), visible due to the electron density asymmetry [32]. (f) Experimental 3D isosurface of TDS in grey scale denoting the distance from $\left(\begin{array}{lll}0 & 0 & 0\end{array}\right)$. Taken from [48]. 

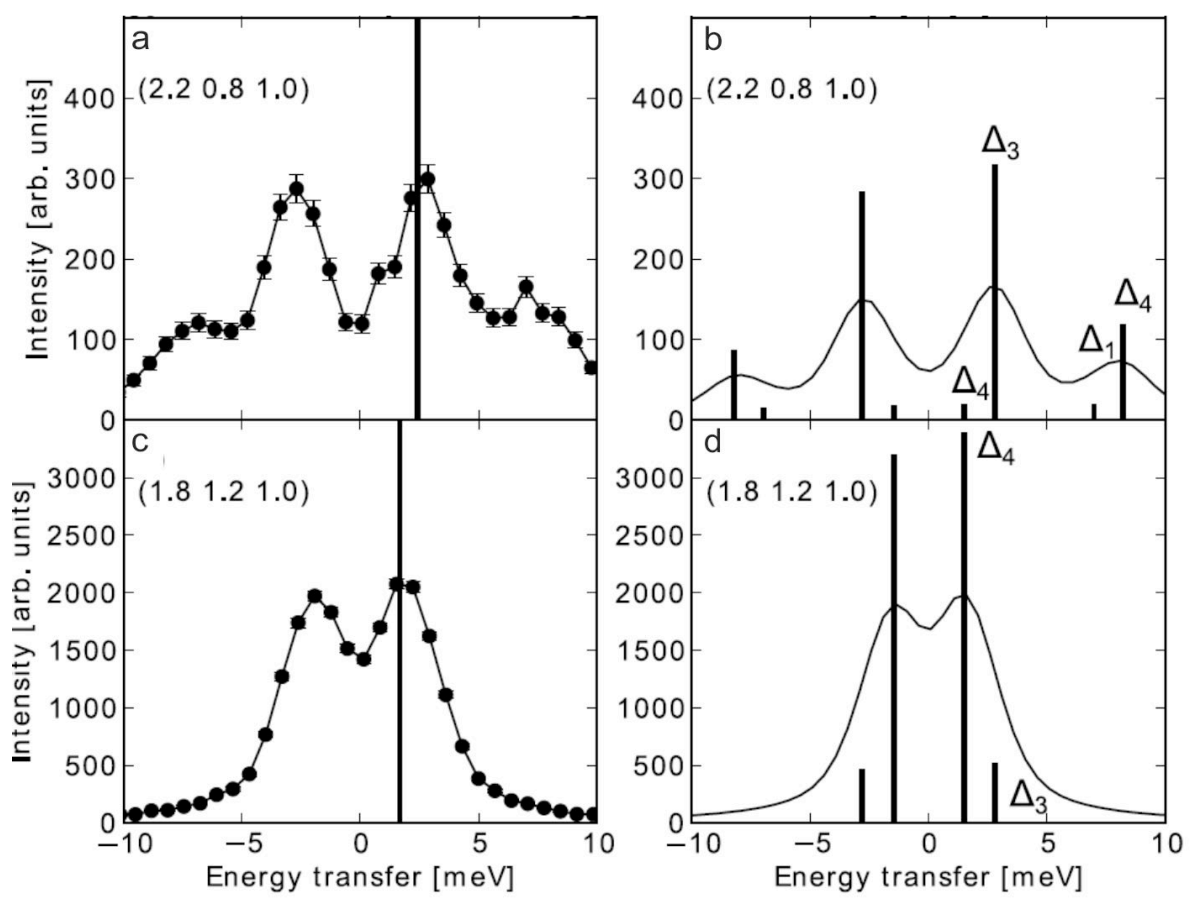

Figure 10. Experimental IXS spectra (left panels) of tin in the HK1 plane. The reduced momentum transfer $\mathbf{q}$ of panels (a) and (c) is equivalent. The peak position of the envelope function of the two acoustic branches is indicated by vertical lines in the experimental spectra. The inelastic contribution of the different branches (vertical lines) as obtained from the DFPT calculation and its convolution with the experimental resolution are shown for the corresponding momentum transfers in the right panels (b) and (d). The vertical lines are scaled with respect to the convoluted spectra for best visualization. Taken from [48].

The similarity of TDS intensity distributions of $\beta$ - and $\gamma$-tin can be appreciated from figure 11. It reflects the symmetry relation of the two phases which have common subgroups, consequently some symmetry elements are retained at the phase transition [49]. The transformation matrix between the two lattices is given by

$$
\left(\begin{array}{l}
a_{\beta} \\
b_{\beta} \\
c_{\beta}
\end{array}\right) \approx\left(\begin{array}{ccc}
-1 & 1 & 0 \\
0 & 0 & 2 \\
1 & 1 & 0
\end{array}\right)\left(\begin{array}{l}
a_{\gamma} \\
b_{\gamma} \\
c_{\gamma}
\end{array}\right),
$$

where the indices indicate the phase. The $\Gamma-\mathrm{H}-\mathrm{M}$ direction in $\beta$-tin for instance corresponds to the $\Gamma-$ $\mathrm{K}-\mathrm{M}$ direction in $\gamma$-tin. The structures of the two phases are different; the momentum transfer dependence of the underlying electronic potential is, however, similar. 


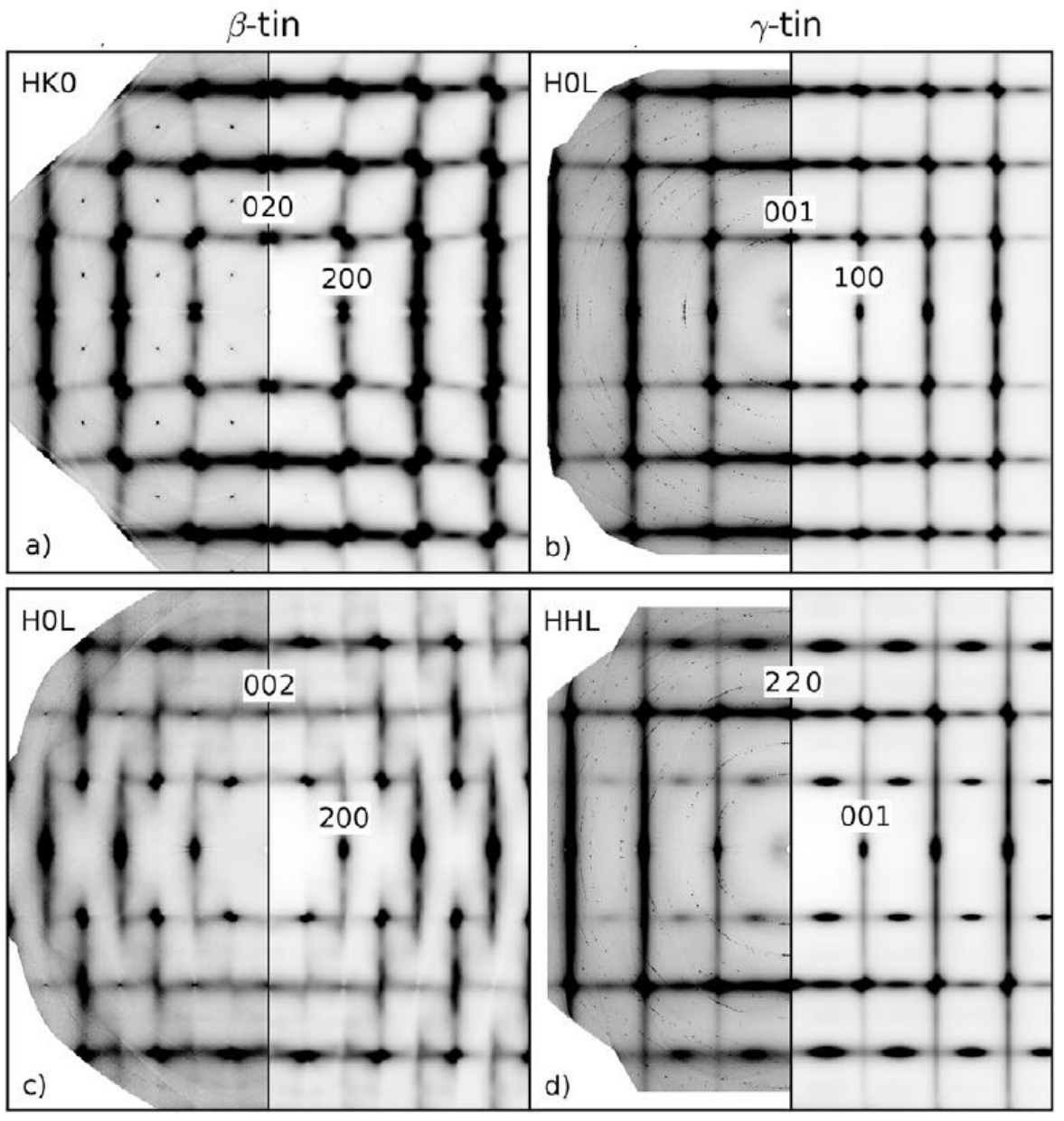

Figure 11. Experimental diffuse scattering (left part of individual panels) and calculated (right part of individual panels) TDS intensity distribution of $\beta$-tin (a) and (c) and $\gamma$-tin (b) and (d) in the indicated reciprocal space sections. The experimental TDS intensity distribution of $\gamma$-tin was obtained from a $\mathrm{Sn}_{0.8} \mathrm{In}_{0.2}$ single crystal, whereas the calculated intensities are for pure $\mathrm{Sn}$. Taken from [48].

\section{Case study: charge density waves in YBCO superconductor}

Recent $\mathrm{x}$-ray scattering experiments revealed strong charge density wave (CDW) correlations in underdoped $\mathrm{YBa}_{2} \mathrm{Cu}_{3} \mathrm{O}_{6-\mathrm{x}}$, one of the most widely studied high-temperature superconductors $[50,51,52]$. Specifically, the CDW was shown to be present throughout the bulk of the material, clearly separated from magnetically ordered states, and competing strongly with superconductivity. As the displacement pattern, corresponding to the CDW, was a priori unknown, the establishment of diffuse scattering «roadmaps» was indispensable for an efficient IXS data collection. Data were recorded at the ID29 ESRF beamline on a needle-like single crystal $(\varnothing 0.03 \mathrm{~mm})$ of underdoped $\mathrm{YBa}_{2} \mathrm{Cu}_{3} \mathrm{O}_{6.6}\left(\mathrm{~T}_{\mathrm{C}}=61 \mathrm{~K}\right)$ in conditions similar to the ones described above. Clear structures related to chain order [53] are visible between the Bragg peaks only in the (HOL) plane and are completely absent in the (0KL) map (figure 12). Since the intensity of these diffuse peaks is about two orders of magnitude larger than those related to the CDW (apparent in the (0KL) plane at low temperature), their total absence in the (0KL) map demonstrates the perfect detwinning of our single crystal [54]. 

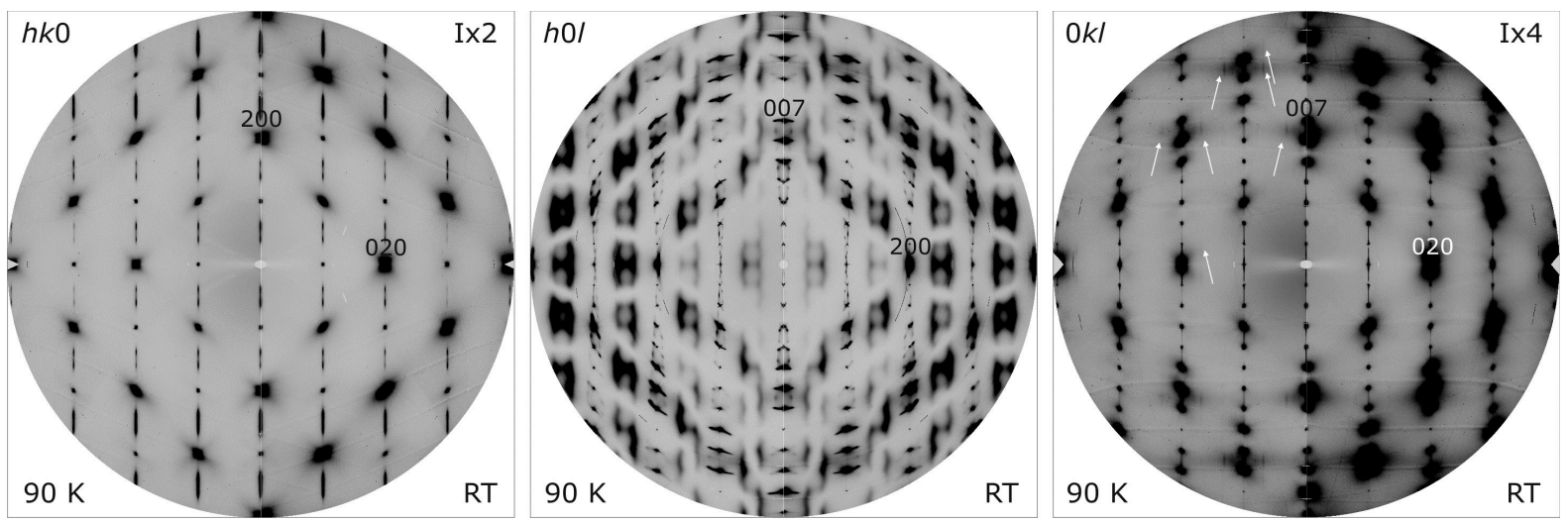

Figure 12. Diffuse scattering maps of high symmetry planes in $\mathrm{YBa}_{2} \mathrm{Cu}_{3} \mathrm{O}_{6.6}$. White arrows indicate the CDW superstructure peaks. The intensity scale is different for the panels as indicated in the top right part of left and right panels. Adapted from [54].

The intensity maps in the $(0 \mathrm{KL})$ plane reveal the emergence of extended features at low temperatures with $\mathrm{q}_{\mathrm{CDW}}=\left(\begin{array}{lll}0 & 0.31 & 0.5\end{array}\right)$ and a significant degree of anisotropy. Similar mappings of the reciprocal space of optimally doped $\mathrm{YBa}_{2} \mathrm{Cu}_{3} \mathrm{O}_{7}$ did not reveal any $\mathrm{CDW}$-like feature at low temperature.

Following the "roadmaps", IXS scans were performed through the (0 0.31 6.5) CDW superstructure peak. For temperatures $\mathrm{T}_{\mathrm{C}}<\mathrm{T}<\mathrm{T}_{\mathrm{CDW}}$, a large broadening of the transverse acoustic and a transverse optical phonon, belonging to the B1 representation (that mostly involve atomic vibrations along the $c$ axis) is observed, together with an intensity increase of the central peak down to $T_{C}$. The resulting dispersion, shown in figure 13, further indicates a strong softening of these two modes across the superconducting transition. These effects occur in a very narrow region of reciprocal space around qCDw.

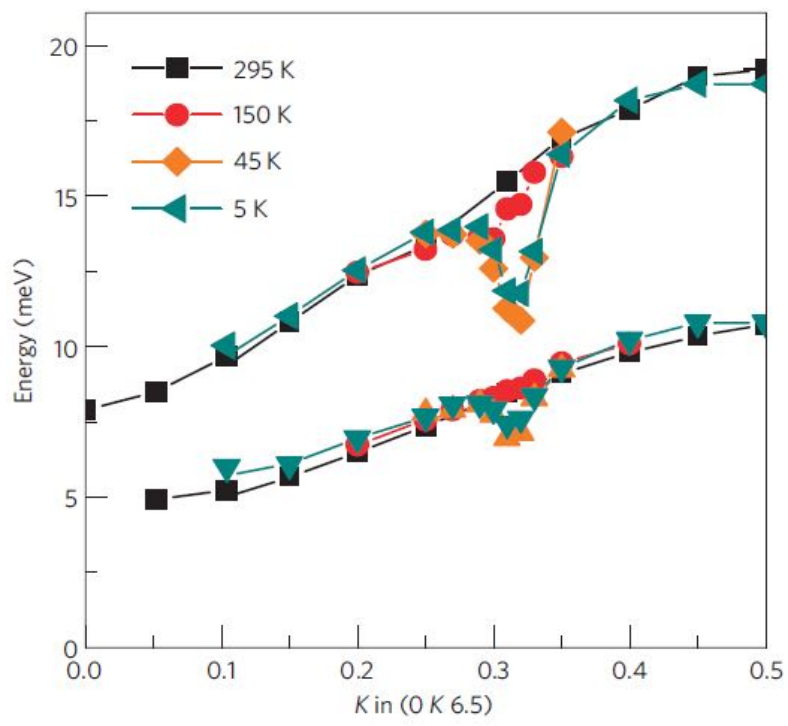

Figure 13. Temperature dependence of the dispersion of the two low-energy phonons along the Z-T direction at $\mathrm{T}=295,150,45$ and $5 \mathrm{~K}$. Adapted from [54]. Color online. 
In-between Bragg reflections: thermal diffuse scattering and vibrational spectroscopy with X-rays

The superconductivity induced renormalization of the phonons is overall large and is a direct signature of the electron-phonon interaction. Due to its very compact localization in momentum space, its contribution to the Cooper pair formation is, however, negligible. Rather, it favors a CDW instability that strongly competes with superconductivity and reduces the superconducting $\mathrm{T}_{\mathrm{C}}$ at moderate doping levels.

\section{Conclusions}

Thermal diffuse scattering experiments can serve as a rigorous benchmark for parameter-free model calculations even for relatively complex structures, in particular if they are complemented with inelastic scattering techniques on powder samples, single crystals or both. Once the validity of the model is established, it then can be used to gain valuable insight into the dynamical properties of materials, often in a more meaningful way than from phonon dispersion curves or phonon-density-ofstates alone.

The acquisition of diffuse scattering roadmaps prior to the inelastic scattering experiment, particularly in the case of non-trivial (i.e. strongly correlated) systems is highly beneficial for choosing the experimental strategy, sometimes providing crucial information and always reducing the necessary measurement time.

Presently, the construction of a diffraction side station on the undulator beamline ID28 at the ESRF is underway. The proposed instrument, operating as a side branch of the IXS spectrometer, shall be primarily dedicated to the study of diffuse scattering in a large class of materials ranging from strongly correlated electron systems to nanoscale-modulated and low-dimensional systems, and will constitute a powerful tool in the study of lattice dynamics complementing the inelastic $\mathrm{x}$-ray scattering studies. Investigations of crystalline systems with correlated disorder will greatly benefit from the dedicated station, thanks to a flexible sample environment. The high brilliance of the x-ray source, coupled to state-of-the-art detection schemes, using a hybrid pixel detector, will open the window to time-resolved studies and studies under extreme conditions of pressure, temperature and magnetic fields. In summary, the diffuse scattering station in combination with the existing IXS instrument on ID28 will constitute a world-wide unique experimental station, offering unprecedented capabilities in the studies of lattice dynamics in condensed matter and of the real structure of functional materials to the large ESRF user community.

\section{Acknowledgments}

The authors would like to acknowledge the courtesy of the Harvard Mineralogical museum and personally the collection curator Dr. Raquel Alonso-Perez for providing the high quality pieces of 
In-between Bragg reflections: thermal diffuse scattering and vibrational spectroscopy with X-rays

natural cristobalite crystals (Cristobalite, HMM\#97849). Authors are grateful to Dr. Clemens SchulzeBriese (SLS, Dectris), Dr. Daniele De Sanctis (ESRF) and Dr. Alexander Popov for providing the beamtime for diffuse scattering studies, Dr. A. Ivanov and Dr. A. Rumiantsev for providing high quality tin single crystals. We are grateful for funding from the German BMBF (project 05K13RF2). 
In-between Bragg reflections: thermal diffuse scattering and vibrational spectroscopy with X-rays

\section{References}

\footnotetext{
${ }^{1}$ Lonsdale K 1948 Crystals and X-rays (G. Bell, London)

${ }^{2}$ Wooster W A 1962 Diffuse X-ray Reflections from Crystals (Clarendon, Oxford)

${ }^{3}$ Olmer P 1948 Acta Cryst. 1 57-63

${ }^{4}$ Holt M, Wu Z, Hong H, Zschack P, Jemian P, Tischler J, Chen H and Chiang T-C 1999 Phys. Rev.
} Lett. $833317-3319$

${ }^{5} \mathrm{Xu}$ R and Chiang C 2005 Z. Kristallogr. 2201009

${ }^{6}$ Strauch D and Dorner B 1986 J. Phys. C: Solid State Phys. 19 2853-2864

${ }^{7}$ Brockhouse B N 1961 Inelastic scattering of neutrons in solids and liquids vol 113 (IAEA, Vienna)

${ }^{8}$ Krisch M and Sette F 2007 Light Scattering in Solids IX, Novel Materials and Techniques, Topics in Applied Physics vol 108 (Springer-Verlag Berlin Heidelberg)

${ }^{9}$ Shvyd'ko Y, Stoupin S, Shu D, Collins S P, Mundboth K, Sutter J and Tolkiehn M 2014 Nature Communications 54219

${ }^{10}$ Bergamin A, Cavagnero G, Mana G and Zosi G 1999 Eur. Phys. J. B 9225

${ }^{11}$ Verbeni R, Sette F, Krisch M H, Bergmann U, Gorges B, Halcoussis C, Martel K, Masciovecchio C, Ribois J F, Ruocco G and Sinn H 1996 J. Synchr. Radiat. 362

${ }^{12}$ De Sanctis D, Beteva A, Caserotto H, Dobias F, Gabadinho J, Giraud T, Gobbo A, Guijarro M, Lentini M, Lavault B, Mairs T, McSweeney S, Petitdemange S, Rey-Bakaikoa V, Surr J, Theveneau P, Leonard G A and Mueller-Dieckmann C 2012 J. Synchrotron Radiat. 19 455-461

${ }^{13}$ Brönnimann C, Eikenberry E F, Henrich B, Johnson I, Mozzanica A, Schlepütz C M, Willmott P R and Schmitt B 2006 J. Synchrotron. Radiat. 13120

${ }^{14}$ Hatsui T and Graafsma H 2015 Int Union Cryst J. 2 371-383

${ }^{15}$ Rigaku Oxford Diffraction

${ }^{16}$ Born M and Huang K 1954 Dynamical theory of Crystal lattices (Oxford University Press, Oxford)

${ }^{17}$ Bosak A and Chernyshov D 2008 Acta Cryst. A 64598

${ }^{18}$ Born M 1942 Rep. Prog. Phys. 9 294-333

${ }^{19}$ Groenewegen P P M and Huiszoon C 1972 Acta Cryst. A28 166-169

${ }^{20}$ Scheringer C 1972 Acta Cryst. A28 512-515

${ }^{21}$ Willis B T M and Pryor A W 1975 Thermal Vibrations in Crystallography (Cambridge University Press)

${ }^{22}$ Buchenau U, Nücker N and Dianoux A J 1984 Phys. Rev. Lett. 532316

${ }^{23}$ Chumakov A I, Monaco G, Fontana A, Bosak A, Hermann R P, BessasD, Wehinger B, Crichton W A, Krisch M, Rüffer R, Baldi G, Carini Jr G, Carini G, D’Angelo G, Gilioli E, Tripodo G, Zanatta M, Winkler B, Milman V, Refson K, Dove M T, Dubrovinskaia N, Dubrovinsky L, Keding R and Yue Y Z 2014 Phys. Rev. Lett. 112025502 
${ }^{24}$ Wehinger B, Bosak A, Refson K, Mirone A, Chumakov A and Krisch M 2015 J. Phys.: Condens. Matter (accepted) (Preprint http://arxiv.org/abs/1501.01196)

${ }^{25}$ Bosak A, Krisch M, Chernyshov D, Winkler B, Milman V, Refson K and Schulze-Briese C 2012 Z. Kristallogr. 22784

${ }^{26}$ Wehinger B, Bosak A, Chumakov A, Mirone A, Winkler B, Dubrovinsky L, Dubrovinskaia N, Brazhkin V, Dyuzheva T and Krisch M 2013 J. Phys.: Condens. Matter 25275401

${ }^{27}$ Clark S J, Segall M D, Pickard C J, Hasnip P J, Probert M J, Refson K and Payne M C 2005 Z. Kristallogr. 220567

${ }^{28}$ Refson K, Tulip P R and Clark S J 2006 Phys. Rev. B 73155114

${ }^{29}$ Bosak A and Chernyshov D 2008 Acta Cryst. A64 598

${ }^{30}$ Dyuzheva T I, Lityagina L M, Bendeliani N A, Nikolaev N A and Dorokhova G I 1998 Crystallogr. Rep. 43 511-3

${ }^{31}$ Dollase W A 1965 Z. Kristallogr. 121369

${ }^{32}$ Dove M T, Pryde A K A, Heine V and Hammonds K D 2007 J. Phys.:

Condens. Matter 19275209

${ }^{33}$ Boysen H, Dorner B, Frey F and Grimm H 1980 J. Phys. C: Solid St. Phys. 136127

${ }^{34}$ Mark K 1995 Meteorite Craters (Tucson, AZ, USA: The University of Arizona Press)

${ }^{35}$ Bosak A, Fischer I, Krisch M, Brazhkin V, Dyuzheva T, Winkler B, Wilson D, Weidner D, Refson K and Milman V 2009 Geophys. Res. Lett. 36, L19309

${ }^{36}$ Dubrovinsky L, Dubrovinskaia N, Saxena S, Tutti F, Rekhi S, Bihan T L, Shen G and Hu J 2001 Chem. Phys. Lett. 333264

${ }^{37}$ Dubrovinsky L S, Dubrovinskaia N A, Prakapenka V, Seifert V, Langenhorst F, Dmitriev V, Weber H P and Le Bihan T 2004 Phys. Earth Planet. Inter. 143-144 231

${ }^{38}$ Kimizuka H, Ogata S and Shibutani Y 2005 Mater. Trans. 461161

${ }^{39}$ Matthias B T, Geballe T H and Compton V B 1963 Superconductivity. Rev. Mod. Phys. 35 1-22

${ }^{40}$ Kubiak R 1986 J. Less-Common Met. 116 307-311

${ }^{41}$ Arlman J J and Kronig R 1943 Physica 10 795-800

${ }^{42}$ Bouman J, Arlman J J and Van Reijen L L 1946 Physica 12 353-70

${ }^{43}$ Prasad S C and Wooster W A1955 Acta Cryst. 8 682-686

${ }^{44}$ Rowe J M 1967 Phys. Rev. 163 547-51

${ }^{45}$ Parratt L G 1967 Proc. R. Soc. A 300 25-44

${ }^{46}$ Rowe J M, Brockhouse B N and Svensson E C 1965 Phys. Rev. Lett. 14 554-6

${ }^{47}$ Ivanov A S, Rumiantsev A Y, Dorner B, Mitrofanov N L and Pushkarev V V 1987 J. Phys. F: Met. Phys. 171925 
In-between Bragg reflections: thermal diffuse scattering and vibrational spectroscopy with X-rays

${ }^{48}$ Wehinger B, Bosak A, Piccolboni G, Refson K, Chernyshov D, Ivanov A, Rumiantsev A and Krisch M 2014 J. Phys. Cond. Matt. 26115401

${ }^{49}$ Raynor G V and Lee J A 1954 Acta Metall. 2 616-20

${ }^{50}$ Ghiringhelli G, Le Tacon M, Minola M, Blanco-Canosa S, Mazzoli C, Brookes N B, De Luca G M, Frano A, Hawthorn D G, He F, Loew T, Moretti Sala M, Peets D C, Salluzzo M, Schierle E, Sutarto R, Sawatzky G A, Weschke E, Keimer B and Braicovich L 2012 Science 337 821-825

${ }^{51}$ Chang J, Blackburn E, Holmes A T, Christensen N B, Larsen J, Mesot J, Liang R, Bonn D A, Hardy W N, Watenphul A, v. Zimmermann M, Forgan E M and Hayden S M 2012 Nature Phys. 8 $871-876$

${ }^{52}$ Achkar A J, Sutarto R, Mao X, He F, Frano A, Blanco-Canosa S, Le Tacon M, Ghiringhelli G, Braicovich L, Minola M, Moretti Sala M, Mazzoli C, Liang R, Bonn D A, Hardy W N, Keimer B, Sawatzky G A and Hawthorn D G 2012 Phys. Rev. Lett. 109167001

${ }^{53}$ V. Zimmermann M, Schneider J R, Frello T, Andersen N H, Madsen J, Käll M, Poulsen H F, Liang R, Dosanjh P and Hardy W N 2003 Phys. Rev. B 68104515

${ }^{54}$ Le Tacon M, Bosak A, Souliou S M, Dellea G, Loew T, Heid R, Bohnen K-P, Ghiringhelli G, Krisch M and Keimer B 2014 Nature Phys. 10 52-58 